Communications in Physics, Vol.22, No. 2 (2012), pp. 125-131

\title{
PHOTON - AXION CONVERSION CROSS-SECTIONS IN A RESONANT CAVITY WITH GENERAL MODE
}

\author{
DAO THI LE THUY AND LE NHU THUC \\ Hanoi National University of Education
}

\begin{abstract}
Photon-axion conversions in the resonant cavity with the general mode (TMmn0) are considered in detail by the Feynman diagram method. The differential cross-sections are presented and numerical evaluations are given. It is shown that there is a resonant conversion for the considered process, in which the conversion cross-sections are much larger than those of the wave guide in the same conditions. Some estimates for experimental conditions are give from our results.
\end{abstract}

\section{INTRODUCTION}

The most attractive candidate for the solution of the strong CP problem is Peccei and Quinn (PQ) mechanism [1], where, the CP-violating phase $\theta\left(\theta \leqslant 10^{-9}\right)$ is explained by the existence of a new pseudo-scalar field, called the axion [2,3]. At present, the axion mass is constrained by laboratory $[4,5]$ and by astrophysical and cosmological considerations $[6,7]$ to between $10^{-6} \mathrm{eV}$ and $10^{-3} \mathrm{eV}$. If the axion has a mass near the low limit of order $10^{-5} \mathrm{eV}$, it is a good candidate for the dark matter of the universe. Besides that, an axino (the fermionic partner of the axion) naturally appears in SUSY models $[8,9]$ which acquires a mass from three-loop Feynman diagrams in a typical range between a few eV up to a maximum of $1 \mathrm{keV}[10,11]$. The candidates for dark matter can appear in different model, in the 3-3-1 models [12-14] or in the supersymmetric and superstring theories [15].

The light particles with a two photon interaction can transform into photons in external electric or magnetic fields by an effect first discussed by Primakoff [16]. This effect is the basis of Sikivie's methods for the detection of axions in a resonant cavity [17]. He suggested that this method can be used to detect the hypothetical galactic axion

flux that would exist if axions were the dark matter of the Universe. Various terrestrial experiments to detect invisible axions by making use of their coupling to photons have been proposed [18-20] and results of such experiments appeared recently [21-23]. The experiment CAST $[24,25]$ at CERN searches for axions from the sun or other sources in the universe. The experiment uses a large magnet from LHC and seaches for exotic particles into axions in the periodic EM field of the resonant cavity.

Consider the conversion of the photon $\gamma$ with momentum q into the axion a with momentum $\mathrm{p}$ in an external electromagnetic field. Using the Feynman rules we get the following expression for the matrix element [26].

$$
\langle p|M| q\rangle=-\frac{g_{a \gamma}}{2(2 \pi)^{2} \sqrt{q_{0} p_{0}}} \epsilon_{\mu}(q, \sigma) \epsilon^{\mu \nu \alpha \beta} q_{\nu} \int_{V} e^{i k r} F_{\alpha \beta}^{c l a s s} d r
$$


where $k \equiv q-p$ is the momentum transfer to the EM field, $g_{a \gamma} \equiv g_{\gamma} \frac{\alpha}{\pi f_{a}}=g_{\gamma} \alpha m_{a}\left(m_{u}+\right.$ $\left.m_{d}\right)\left[\pi f_{\pi} m_{\pi} \sqrt{m_{u} m_{d}}\right]^{-1}$ and $\epsilon^{\mu}(q, \sigma)$ represents the polarization vector of the photon.

Expression (1) is valid for an arbitrary external EM field. In the following we shall use it for the case, namely conversions in the periodic EM field of the resonant cavity with $T M_{m n 0}$ mode. Here we use the following notations: $q \equiv|q|, p \equiv|p|=\left(p_{0}^{2}-m_{a}^{2}\right)^{1 / 2}$ and $\theta$ is the angle between $\mathrm{p}$ and $\mathrm{q}$.

\section{CONVERSION IN A RESONANT CAVITY WITH GENERAL MODE}

For the sake of simplicity we choose the lowest nontrivial solution of the resonant cavity, namely the $T M_{m n 0}$ mode $[27,28]$

$$
\begin{gathered}
E_{z}=E_{0} \sin \left(\frac{m \pi x}{a}\right) \sin \left(\frac{n \pi y}{b}\right), \\
H_{x}=\frac{i \epsilon \pi}{\omega b} E_{0} \sin \left(\frac{m \pi x}{a}\right) \cos \left(\frac{n \pi y}{b}\right), \\
H_{y}=-\frac{i \epsilon \pi}{\omega a} E_{0} \cos \left(\frac{m \pi x}{a}\right) \sin \left(\frac{n \pi y}{b}\right)
\end{gathered}
$$

here the propagation of the EM wave is in the z-direction.

Substitution of (2) into (1) gives us the following expression for the matrix element

$$
\langle p|M| q\rangle=-\frac{g_{a \gamma}}{(2 \pi)^{2} \sqrt{q_{0} p_{0}}}\left[\left(\epsilon_{1}(\mathbf{q}, \tau) q_{2}-\epsilon_{2}(\mathbf{q}, \tau) q_{1}\right) F_{z}+\epsilon_{1}(\mathbf{q}, \tau) q_{0} F_{x}+\epsilon_{2}(\mathbf{q}, \tau) q_{0} F_{y}\right]
$$

where $p_{0} \equiv q_{0}+\omega, \vec{k}=\vec{q}-\vec{p}$ and

$$
\begin{aligned}
& F_{z}=-\frac{8 E_{0}}{a b}\left(a\left(q_{x}-p_{x}\right) \cos \frac{a\left(q_{x}-p_{x}\right)}{2} \sin \frac{m \pi}{2}-m \pi \cos \frac{m \pi}{2} \sin \frac{a\left(q_{x}-p_{x}\right)}{2}\right) \times \\
&\left(b\left(q_{y}-p_{y}\right) \cos \frac{b\left(q_{y}-p_{y}\right)}{2} \sin \frac{n \pi}{2}-n \pi \cos \frac{n \pi}{2} \sin \frac{b\left(q_{y}-p_{y}\right)}{2}\right) \sin \frac{d\left(q_{z}-p_{z}\right)}{2} \times \\
& {\left[\left(\frac{m^{2} \pi^{2}}{a^{2}}-\left(q_{x}-p_{x}\right)^{2}\right)\left(\frac{n^{2} \pi^{2}}{b^{2}}-\left(q_{y}-p_{y}\right)^{2}\right)\left(q_{z}-p_{z}\right)\right]^{-1} } \\
& F_{x}=-\frac{8 \pi \epsilon E_{0}}{\omega a b^{2}}\left(a\left(q_{x}-p_{x}\right) \cos \frac{a\left(q_{x}-p_{x}\right)}{2} \sin \frac{m \pi}{2}-m \pi \cos \frac{m \pi}{2} \sin \frac{a\left(q_{x}-p_{x}\right)}{2}\right) \times \\
&\left(n \pi \sin \frac{n \pi}{2} \cos \frac{b\left(q_{y}-p_{y}\right)}{2}-b\left(q_{y}-p_{y}\right) \sin \frac{b\left(q_{y}-p_{y}\right)}{2} \cos \frac{n \pi}{2}\right) \sin \frac{d\left(q_{z}-p_{z}\right)}{2} \times \\
& {\left[\left(\frac{m^{2} \pi^{2}}{a^{2}}-\left(q_{x}-p_{x}\right)^{2}\right)\left(\frac{n^{2} \pi^{2}}{b^{2}}-\left(q_{y}-p_{y}\right)^{2}\right)\left(q_{z}-p_{z}\right)\right]^{-1} }
\end{aligned}
$$




$$
\begin{aligned}
& F_{y}= \frac{8 \pi \epsilon E_{0}}{\omega a^{2} b}\left(m \pi \cos \frac{a\left(q_{x}-p_{x}\right)}{2} \sin \frac{m \pi}{2}-a\left(q_{x}-p_{x}\right) \cos \frac{m \pi}{2} \sin \frac{a\left(q_{x}-p_{x}\right)}{2}\right) \times \\
&\left(b\left(q_{y}-p_{y}\right) \cos \frac{b\left(q_{y}-p_{y}\right)}{2} \sin \frac{n \pi}{2}-n \pi \cos \frac{n \pi}{2} \sin \frac{b\left(q_{y}-p_{y}\right)}{2}\right) \sin \frac{d\left(q_{z}-p_{z}\right)}{2} \times \\
& {\left[\left(\frac{m^{2} \pi^{2}}{a^{2}}-\left(q_{x}-p_{x}\right)^{2}\right)\left(\frac{n^{2} \pi^{2}}{b^{2}}-\left(q_{y}-p_{y}\right)^{2}\right)\left(q_{z}-p_{z}\right)\right]^{-1} }
\end{aligned}
$$

where $\mathrm{a}, \mathrm{b}$ and $\mathrm{d}$ are three dimensions of the cavity, $\omega$ is the frequency of the EM field and $\mathrm{m}, \mathrm{n}=0,1,2,3 \ldots$ Substituting Eq. (4) into Eq. (3) we finally obtain the DCS for conversions

$$
\begin{gathered}
\frac{d \sigma}{d \Omega}=\frac{1}{2} \frac{g_{a \gamma}^{2}}{(2 \pi)^{2}} \frac{p_{0}}{q_{0}}\left[\left(q_{x}^{2}+q_{y}^{2}\right) F_{z}^{2}+\left(1-\frac{q_{x}^{2}}{q^{2}}\right) q_{0}^{2} F_{x}^{2}+\left(1-\frac{q_{y}^{2}}{q^{2}}\right) q_{0}^{2} F_{y}^{2}\right. \\
\left.-2 q_{x} q_{y} F_{x} F_{y}-2 q_{0} q_{x} F_{y} F_{z}+2 q_{0} q_{y} F_{x} F_{z}\right] .
\end{gathered}
$$

In the first case, we assume that the momentum of the photon is parallel to the z-axis, we have $q=q_{z}$ and $q_{x}=q_{y}=0$ (the direction of the EM field). From the Eq. (5) we obtain the DCS for conversions for this case

$$
\frac{d \sigma}{d \Omega^{\prime}}=\frac{1}{2} \frac{g_{a \gamma}^{2}}{(2 \pi)^{2}} p_{0} q_{0}\left[F_{x}^{2}+F_{y}^{2}\right]=\frac{1}{2} \frac{g_{a \gamma}^{2}}{(2 \pi)^{2}} q^{2}\left(1+\frac{\omega}{q}\right)\left[F_{x}^{2}+F_{y}^{2}\right]
$$

where

$$
\begin{aligned}
& F_{x}=-\frac{8 \pi \epsilon E_{0}}{\omega a b^{2}}(\left.-a p_{x} \cos \frac{a p_{x}}{2} \sin \frac{m \pi}{2}+m \pi \cos \frac{m \pi}{2} \sin \frac{a p_{x}}{2}\right) \times \\
&\left(-b p_{y} \cos \frac{n \pi}{2} \sin \frac{b p_{y}}{2}+n \pi \sin \frac{n \pi}{2} \cos \frac{b p_{y}}{2}\right) \sin \frac{d\left(q-p_{z}\right)}{2} \times \\
& {\left[\left(\frac{m^{2} \pi^{2}}{a^{2}}-p_{x}^{2}\right)\left(\frac{n^{2} \pi^{2}}{b^{2}}-p_{y}^{2}\right)\left(q-p_{z}\right)\right]^{-1}, }
\end{aligned}
$$

and

$$
\begin{aligned}
& F_{y}=\frac{8 \pi \epsilon E_{0}}{\omega a^{2} b}\left(-a p_{x} \cos \frac{m \pi}{2} \sin \frac{a p_{x}}{2}+m \pi \cos \frac{a p_{x}}{2} \sin \frac{m \pi}{2}\right) \times \\
&\left(-b p_{y} \cos \frac{b p_{y}}{2} \sin \frac{n \pi}{2}+n \pi \cos \frac{n \pi}{2} \sin \frac{b p_{y}}{2}\right) \sin \frac{d\left(q-p_{z}\right)}{2} \times \\
& {\left[\left(\frac{m^{2} \pi^{2}}{a^{2}}-p_{x}^{2}\right)\left(\frac{n^{2} \pi^{2}}{b^{2}}-p_{y}^{2}\right)\left(q-p_{z}\right)\right]^{-1} . }
\end{aligned}
$$

In the sphere coordinate, we have $p_{x}=p \sin \theta \cos \varphi^{\prime} ; p_{y}=p \sin \theta \sin \varphi^{\prime}$ and $p_{z}=p \cos \theta$, where $\varphi^{\prime}$ is the angle between the $\mathbf{x}$-axis and the projection of $\mathbf{p}$ on the xy-plane and $d \Omega^{\prime}=d \varphi^{\prime} d \cos \theta$. From Eq. (6), Eq. (7) and Eq. (8) it is easy to show that there are no conversions when $\theta=0$ and $\varphi^{\prime}=\frac{\pi}{2}$. When $\theta=\frac{\pi}{2}$ and $\varphi^{\prime}=0$ and in the limit $q \rightarrow \frac{\pi}{d}$, we get the DCS for conversions 


$$
\frac{d \sigma}{d \Omega^{\prime}}=\frac{8 g_{a \gamma}^{2} E_{0}^{2}}{a^{2} n^{2} \pi^{2} \omega^{2}} \frac{1}{\left(\frac{m^{2} \pi^{2}}{a^{2}}-p^{2}\right)^{2}}\left(1+\frac{\omega d}{\pi}\right)\left\{-a p \cos \frac{a p}{2} \sin \frac{m \pi}{2}+m \pi \cos \frac{m \pi}{2} \sin \frac{a p}{2}\right\}^{2} \sin ^{2} \frac{n \pi}{2}
$$

Next, if the momentum of the photon is perpendicular to the direction of the EM field, i.e. in the y-axis, we have $q=q_{y}$ and $q_{x}=q_{z}=0$. From the Eq. (5) we obtain the DCS for conversions for this case

$$
\frac{d \sigma}{d \Omega^{\prime \prime}}=\frac{1}{2} \frac{g_{a \gamma}^{2}}{(2 \pi)^{2}} p_{0} q_{0}\left[F_{x}^{2}+F_{z}^{2}\right]=\frac{1}{2} \frac{g_{a \gamma}^{2}}{(2 \pi)^{2}} q^{2}\left(1+\frac{\omega}{q}\right)\left[F_{x}^{2}+F_{z}^{2}\right]
$$

where

$$
\begin{aligned}
& F_{z}=-\frac{8 E_{0}}{a b}\left(-a p_{x} \cos \frac{a p_{x}}{2} \sin \frac{m \pi}{2}+m \pi \cos \frac{m \pi}{2} \sin \frac{a p_{x}}{2}\right) \times \\
&\left(b\left(q-p_{y}\right) \cos \frac{b\left(q-p_{y}\right)}{2} \sin \frac{n \pi}{2}-n \pi \cos \frac{n \pi}{2} \sin \frac{b\left(q-p_{y}\right)}{2}\right) \sin \frac{d p_{z}}{2} \times \\
& \quad\left[\left(\frac{m^{2} \pi^{2}}{a^{2}}-p_{x}^{2}\right)\left(\frac{n^{2} \pi^{2}}{b^{2}}-\left(q-p_{y}\right)^{2}\right) p_{z}\right]^{-1}
\end{aligned}
$$

and

$$
\begin{aligned}
F_{x}=-\frac{8 \pi \epsilon E_{0}}{\omega a b^{2}}\left(-a p_{x} \cos \frac{a p_{x}}{2} \sin \frac{m \pi}{2}+m \pi \cos \frac{m \pi}{2} \sin \frac{a p_{x}}{2}\right) \times \\
\left(n \pi \sin \frac{n \pi}{2} \cos \frac{b\left(q-p_{y}\right)}{2}-b\left(q-p_{y}\right) \sin \frac{b\left(q-p_{y}\right)}{2} \cos \frac{n \pi}{2}\right) \sin \frac{d p_{z}}{2} \times \\
{\left[\left(\frac{m^{2} \pi^{2}}{a^{2}}-p_{x}^{2}\right)\left(\frac{n^{2} \pi^{2}}{b^{2}}-\left(q-p_{y}\right)^{2}\right) p_{z}\right]^{-1} }
\end{aligned}
$$

In the sphere coordinate, we have $p_{x}=p \sin \theta \cos \varphi^{\prime \prime} ; p_{y}=p \cos \theta$ and $p_{z}=p \sin \theta \sin \varphi^{\prime \prime}$, where $\varphi^{\prime \prime}$ is the angle between the z-axis and the projection of $\mathbf{p}$ on the xz-plane and $d \Omega^{\prime \prime}=d \varphi^{\prime \prime} d \cos \theta$. From Eq. (10), Eq. (11) and Eq. (12) it is easy to show that there are no conversions when $\theta=\varphi^{\prime \prime}=0$. When $\theta=\varphi^{\prime \prime}=\frac{\pi}{2}$ and in the limit $p \rightarrow \frac{m \pi}{a}$, we get the DCS for conversions

$$
\begin{aligned}
& \frac{d \sigma}{d \Omega^{\prime \prime}}=\frac{1}{2} \frac{g_{a \gamma}^{2} E_{0}^{2} d^{2} a^{2}}{\pi^{2} \omega^{2} m^{2}(2 \pi)^{2}\left(\frac{n^{2} \pi^{2}}{b^{2}}-q^{2}\right)^{2}} q^{2}\left(1+\frac{\omega}{q}\right)\{m \pi-\sin m \pi\}^{2} \\
& \quad\left[\omega q \cos \frac{b q}{2} \sin \frac{n \pi}{2}-\frac{n \pi \omega}{b} \sin \frac{b q}{2} \cos \frac{n \pi}{2}+\frac{\pi^{2}}{b}\left(n \cos \frac{b q}{2} \sin \frac{n \pi}{2}-\frac{b q}{\pi} \cos \frac{n \pi}{2} \sin \frac{b q}{2}\right)\right]^{2}
\end{aligned}
$$

From Eq. (14), for each couple value of $m$ and $n$ we have the DCS for each conversion. In each case, the DCS depends quadratically on the amplitude $E_{0}$, photon momentum $q$ and two dimensions of the resonant cavity. 


\section{DISCUSSION AND CONCLUSION}

(a) From Eq. (13) we have the DCS for conversion with the lowest TM mode $\left(T M_{110}\right)[26]$. Note that there is no E-wave of types (000), (001), (010), (100), (101) and (011).

$$
\frac{d \sigma}{d \Omega^{\prime \prime}}=\frac{1}{2} \frac{g_{a \gamma}^{2} E_{0}^{2} d^{2} a^{2}}{\omega^{2} m^{2}(2 \pi)^{2}\left(\frac{\pi^{2}}{b^{2}}-q^{2}\right)^{2}} q^{2}\left(1+\frac{\omega}{q}\right)\left(\omega q+\frac{\pi^{2}}{b^{2}}\right)^{2} \cos ^{2} \frac{b q}{2} .
$$

To compare the results with the wave guide (for details, see Ref. 20) we can introduce the DCS's ration $\mathrm{R}$ between the $T M_{110}$ mode of the resonant cavity and the $T E_{10}$ mode of the wave guide (for the best case)

$$
R=\frac{(D C S)_{110}}{(D C S)_{10}} \sim \frac{q^{2}}{\omega^{2}}\left(1+\frac{\omega}{q}\right)^{2} .
$$

From Eq. (15), in the limit $\omega^{2}=m_{a}^{2} \ll q^{2}$, we have $(D C S)_{110} \gg(D C S)_{10}$. This means that the conversion cross-sections of the resonant cavity are much larger than those of the wave guide in the same conditions.

(b) From Eq. (13) the DCS for conversion with the $T M_{1 n 0}, T M_{2 n 0}, T M_{3 n 0}, T M_{4 n 0}, \ldots$ modes $(n=1,2,3 \ldots)$ are evaluated in detail and for each value of $\mathrm{n}$ and for all values of $\mathrm{m}$, the DCS's have the same value. We estimate the result in C.G.S. units, by using data as in Refs. 17 and $20, E_{0}=10^{6} \mathrm{~cm}^{-1 / 2} g^{1 / 2} s^{-1}, a=b=d=100 \mathrm{~cm}$ and $\omega=m_{a}=10^{-6} \mathrm{eV}$. When $m=1$ and $n=1,2,3,4,5, \ldots$ the DCS for Eq. (13) as a function of the momentum of photon were plotted in Fig. 1. We can see from the figure, the momentum of the photons is perpendicular to the momentum of axion, when the value of n's is more high, then DCS of the resonant conversion are more large and move to region of high values of the momentum $q$. With each value of $n$ and at the high value of the momentum q, DCS's have very small values. This is useful for experiment to detect axion.

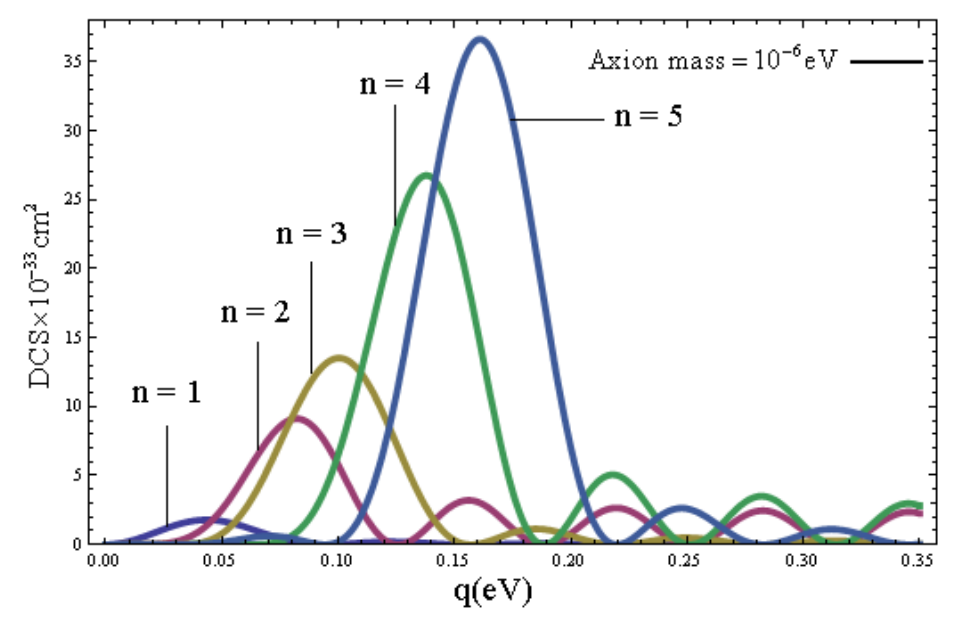

Fig. 1. 


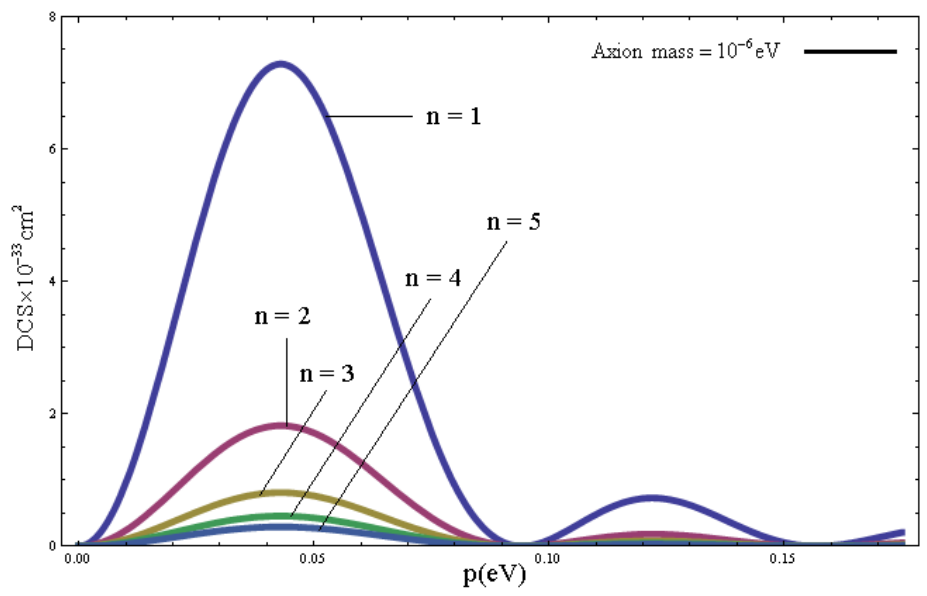

Fig. 2.

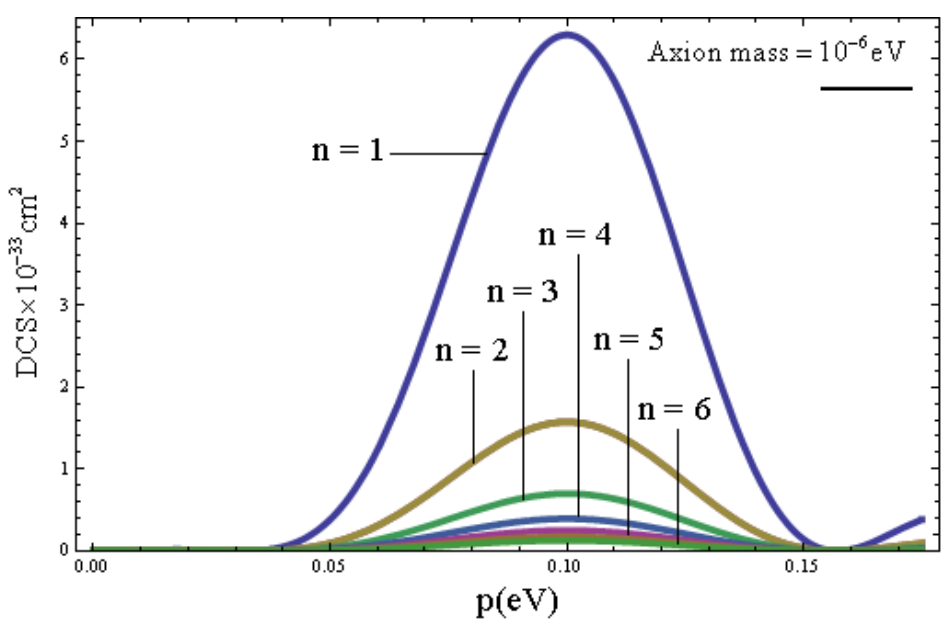

Fig. 3.

When the momentum of photon is parallel to the z-axis (the direction of the EM field): For value of $\mathrm{m}$ is even, there are no conversion. For value of $m$ is odd and $n=$ $1,2,3,4,5 \ldots$, the result of the DCS in C.G.S. units is estimated. When $\mathrm{m}=1$ and $\mathrm{n}=1,2$, $3,4,5 \ldots$, by using data as in Refs. 17 and $20, E_{0}=10^{6} \mathrm{~cm}^{-1 / 2} \mathrm{~g}^{1 / 2} \mathrm{~s}^{-1} a=b=d=100 \mathrm{~cm}$ and $\omega=m_{a}=10^{-6} \mathrm{eV}$, the DCS for Eq. (9) as a function of the momentum of axion were plotted in Fig. 2. From Fig. 2, we can see, there only exist the resonant conversion at the value $p \simeq 4.7 \times 10^{-2} \mathrm{eV}$ and the DCS for conversion with the lowest TM mode is largest (in the case $m=1$ and $n=1$ ).

Similarity, for $m=3,5,7, \ldots$ then the DCS for conversion is largest with the lowest TM mode and maximum of the conversion moves to the high value of the momentum $p$ (see Fig. 3 with $m=3$ ). 


\section{REFERENCES}

[1] R. D Peccei and H. R.Quinn, Phys. Rev. 38 (1977) 1440.

[2] S.Wieinberg,Phys. Lett. 40 (1977) 223.

[3] F. Wilczek, Phys. Rev. Lett. 40 (1977) 279.

[4] J. E. Kim, Phys. Rep. 150 (1987) 1

[5] R. D Peccei, in CP Violation, Advanced Series on Directions in Higg Energy Physics, Vol. 3, ed. C. Jarlskog (World Scientific, 1989).

[6] M. S. Turner, Phys. Rep. 197 (1990) 67.

[7] G. G. Raffelt, Phys. Rep. 198 (1990) 1.

[8] I. E. Kim, Phys. Lett. B136 (1984) 387.

[9] K. Rajagopal, M. S. Turner and F. Wilczek, Nucl. Phys. B358 (1991) 447.

[10] M. I. Vysotsky and M. B. Voloshin, Yad. Fiz. Rev. 44 (1986) 845.

[11] J. E. Kim, A. Masiero and D. V. Nanopoulos, Phys. Lett. B139 (1984) 346.

[12] D. Fregolente and M. D. Tonasse, Phys. Lett. B555 (2003) 7.

[13] H. N. Long and N. Q. Lan, Europhys. Lett. 64 (2003) 571.

[14] A. G. Dias, V. P. Pleitez and M. Tonasse, Phys. Rev. D67 (2003) 095008.

[15] K. S. Babu, I. Gogoladze and K. Wang, Phys. Lett. B560 (2003) 214.

[16] H. Primakoff, Phys. Rev. 81 (1951) 899.

[17] P. Sikivie, Phys. Rev. D32 (1985) 2988.

[18] K. Van Bibber, N. R. Dagdeviren, S. E. Koomin, A. K. Kerman and H. N. Nelson, Phys. Rev. Lett. 59 (1987) 759.

[19] H. N. Long, D. V. Soa and Tuan. A. Tran, Phys. Lett. B357 (1995) 469.

[20] D. V. Soa and H. H. Bang, Int. J. Mod. Phys. A16 (2001) 1491.

[21] C. Hagman, P. Sikivie, N. S. Sullivan and D. B. Tanner, Phys. Rev. D42 (1990) 1297.

[22] S. Moriyama, M. Minowa, T. Manba, Y. Inoue, Y. Takasu and A. Yamamoto, Phys. Lett. B434 (1998) 174 .

[23] S. Asztalos et al., Phys. Rev. D64 (2001) 092003.

[24] I. G. Irastorza et al., Nucl. Phys. Proc. Suppl. 114 (2003) 75.

[25] S. Aune et al., Nucl. Instrum. Meth. A604 (2009) 15.

[26] D. V. Soa, H. N. Long and L. N. Thuc, Mod. Phys. Lett. A22 (2007) 19.

[27] J. D. Jackson, Classical Electrodynamics (Dover, 1975).

[28] H. R. L. Lamont, Wave Guides (Methuen, 1942). 